\title{
Estimativas de Parâmetros de Curvas de Crescimento de Bovinos Zebu, Criados no Estado de Pernambuco ${ }^{1}$
}

\author{
Kleber Régis Santoro², Severino Benone Paes Barbosa ${ }^{3}$, Lúcia Helena de Albuquerque Brasil ${ }^{4}$, \\ Eufrázio de Souza Santos ${ }^{5}$
}

\begin{abstract}
RESUMO - Foram analisados dados de pesagem constantes no banco de dados de Controle de Desenvolvimento Ponderal da Associação Brasileira de Criadores de Zebu (ABCZ), referentes a 24.028 animais Zebu das raças Guzerá, Nelore e Nelore Mocho, nascidos entre 1960 e 2000, objetivando-se estimar parâmetros de curvas de crescimento por meio de equações não-lineares e verificar a influência de efeitos fixos e aleatórios sobre as estimativas. As pesagens ocorreram ao nascimento e em intervalos de 90 dias até dois anos de idade. Para análise dos dados de peso-idade, foram utilizados os seguintes modelos não-lineares: Brody, Gompertz, Logístico, Mitscherlich, von Bertalanffy, Richards e Logístico duplo. As fontes de variação estudadas no modelo misto foram sexo, propriedade, ano e mês de nascimento, tipo de sistema de criação e interação sexo*sistema de criação, como efeitos fixos, e reprodutor e mãe, como efeitos aleatórios. A equação Logística Dupla foi a que apresentou o melhor ajustamento e a Mitscherlich, o pior, segundo o número de convergências e o índice de ajuste. Os efeitos fixos mais influentes foram sexo, propriedade, ano e mês de nascimento e sistema de criação. O efeito aleatório mais influente foi o de mãe. A variabilidade encontrada pode possibilitar a exploração da modificação da curva de crescimento por meio de programas de melhoramento genético.
\end{abstract}

Palavras-chave: Guzerá, modelo misto, modelos não-lineares, Nelore, Nelore Mocho, zebu

\section{Growth Curve Parameters for Zebu Breeds Raised at Pernambuco State, Northeastern Brazil}

\begin{abstract}
Weight records of 24.028 zebu animals from Guzerá, Nelore, and Polled Nelore breeds available from Brazilian Association of Zebu Breeders (ABCZ) database were used to estimate growth curve parameters. The measures were taken on animals which were born between 1960 and 2000, every three months, from birth to 24 months of age. Non-linear Brody, Gompertz, Logistic, Mitscherlich, von Bertalanffy, Richards, and Double Logistic models including sex, farm, year of birth, month of birth, raising system, and interaction sex*raising system as fixed effects and sire and dam, as random effects were compared. Considering the number of iterations for convergence and the adjustment criteria, Double Logistic was the best fitting model and the Mitscherlich was the worst one. The most important fixed effects were sex, farm, year of birth, month of birth and raising system. The major random effect was the dam effect. The variability observed in this study indicates the possibility of changing the growth curve by a breeding program.
\end{abstract}

Key Words: Guzerá, mixed model, Nelore, Nelore Mocho, non-linear models, zebu

\section{Introdução}

O processo de crescimento de um organismo pode ser visto, de uma forma geral, como uma resposta completa à interação entre os processos de catabolismo e anabolismo, sujeitos às influências do meio, às características genéticas do indivíduo e à interação genótipo-ambiente (Trenkle \& Marple, 1983).

Pesquisadores têm se dedicado em descrever as medidas tomadas sucessivamente no tempo, por meio de modelos, considerando esses dados separadamente ou como pontos em um processo contínuo.
Estas medidas, denominadas longitudinais, não se referem apenas às características contínuas, mas também às discretas.

Entre as medidas de crescimento corporal animal possíveis de utilização, uma das mais comuns, que não altera o organismo sob análise e que pode ser mensurada a baixo custo é o peso em determinadas idades (Lawrence \& Fowler, 1997; Pearson \& Dutson, 1991). Dados referentes a essas medidas distribuemse ao longo do tempo de forma semelhante a curvas exponenciais e podem ser analisados por modelos que consideram relações não-lineares de peso e idade.

\footnotetext{
${ }^{1}$ Parte da tese de doutorado do primeiro autor financiada pelo CNPq

2 Professor - UAG/UFRPE. Rua Ernesto Dourado, 82 - Bairro Heliópolis - CEP: 55296-190 - Garanhuns - PE. E.mail: ksantoro@ufrpe.br 3 Professor DZ/UFRPE. Rua Dom Manoel de Medeiros, s/n; Bairro de Dois Irmãos - CEP: 52171-900, Recife - PE. E.mail: sbarbosa@ufrpe.br ${ }^{4}$ Professora DZ/UFRPE. Rua Dom Manoel de Medeiros, s/n; Bairro de Dois Irmãos - CEP: 52171-900, Recife - PE.

5 Professor Pós-Graduação em Biometria/UFRPE. Rua Dom Manoel de Medeiros, s/n; Bairro de Dois Irmãos - CEP: 52171-900, Recife - PE.
} 
As equações da família de Richards têm sido amplamente utilizadas na análise de dados de crescimento de bovinos de corte e de leite (Brown et al., 1976; Fitzhugh Jr., 1976; Goonewardene et al., 1981; Ludwig et al., 1981; Nobre et al., 1987; Kroll \& Tornero, 1994; Perotto et al., 1994; Koenen \& Groen, 1996; Oliveira et al., 2000; Mazzini, 2001).

Entre os problemas da abordagem pelos modelos não-lineares da família de Richards, destaca-se a nãoconvergência (Nobre et al., 1987), os parâmetros estimados fora de limites plausíveis biologicamente (Ludwig et al., 1981) e a desconsideração da heterogeneidade da variância para as medidas, da correlação entre as medidas e da autocorrelação dos erros (Mazzini, 2001). Alguns autores propuseram extensões aos modelos da família de Richards como forma de melhorar a qualidade do ajuste: Kroll \& Tornero (1994), que consideraram erros autorregressivos e efeito da estação do ano; Koenen \& Groen (1996), que empregaram a função logística em duas fases, a qual tem a vantagem de estimar diretamente os parâmetros nos dois pontos de inflexão da curva de crescimento; e Mazzini (2001), que analisou dados considerando heterogeneidade de variância e autocorrelação nos erros.

Os benefícios do uso desses modelos no melhoramento genético animal seriam a estimação dos parâmetros para as curvas e a identificação dos animais mais apropriados a determinados fins, como maior ganho de peso em uma fase específica da vida. Além dos parâmetros analisáveis diretamente da equação de Richards ( $a, k$ e $m$ ) e que possuem interpretação biológica, segundo Perotto et al. (1997), podem ser deduzidos os seguintes índices no auxílio ao melhoramento genético: taxa média de crescimento absoluto, taxa média de maturação absoluta, peso, grau de maturidade e idade no ponto de inflexão, máxima velocidade de ganho de peso e máxima taxa de maturação.

Estudos da influência de efeitos fixos e aleatórios sobre os parâmetros podem auxiliar na interpretação dos fatores que afetam a característica, promovendo melhor análise e auxiliando o planejamento de programas de melhoramento genético.

As funções pertencentes à família de Richards foram agrupadas por Richards (1959), que agregou algumas funções de crescimento já conhecidas e utilizadas na análise de dados individuais de animais e plantas e de crescimento de populações, em uma única função flexível da qual poderiam ser deduzidas. Entre os casos especiais da família de Richards, encontram-se as funções de Brody ou Monomolecular (Brody, 1945), Logística ou Autocatalítica (Nelder 1961), von Bertalanffy (von Bertalanffy, 1957) e Gompertz (Winsor, 1932), com os parâmetros $a, b$ e $k$, e a de Richards (Richards, 1959), com um parâmetro a mais, o $\mathrm{m}$. A função geral, neste caso, é dada por:

$$
Y_{t}=a\left(1 \pm b e^{-k t}\right)^{m}
$$

em que, segundo Fitzhugh Jr. (1976) e Nobre et al. (1987), $Y_{t}$ é o peso corporal na idade $t$; $a$, o valor assintótico, interpretado como peso assintótico ou peso à maturidade; $b$, um parâmetro escala (constante de integração) que não tem interpretação biológica; $k$, o índice de maturidade ou de precocidade; $k^{-1}$, tempo para atingir a maturidade; $m$, o parâmetro de inflexão, que estabelece o grau de maturidade $u_{I}$, no ponto de inflexão $u_{I}=[(m-1) / m]^{m}$, de modo que, de forma generalizada, $u_{t}$ é a proporção peso/idade $t: u_{t}=Y_{t} / a=\left(1 \pm b e^{-k t}\right)^{m}$. O sinal inferior de \pm deve ser aplicado quando $m \geq 1$ e o superior, quando $m<0$.

Os objetivos neste trabalho foram estimar parâmetros de modelos não-lineares de curvas de crescimento de bovinos Zebu no estado de Pernambuco, determinar o modelo mais adequado para a descrição das observações e verificar a influência de efeitos fixos e aleatórios sobre esses parâmetros.

\section{Material e Métodos}

Foram utilizados dados constantes no banco de dados de Controle de Desenvolvimento Ponderal da Associação Brasileira de Criadores de Zebu (ABCZ), referentes a 24.028 animais Zebu (4.436 Guzerá, 14.035 Nelore e 5.557 Nelore Mocho) nascidos entre 1960 e 2000 e criados no estado de Pernambuco.

Os animais foram criados a pasto, semiestabulados, estabulados ou com mudança no sistema em algum período de criação. As pesagens ocorreram ao nascimento e em intervalos de 90 dias até dois anos de idade, totalizando nove pontos amostrais.

Os seguintes modelos não-lineares foram utilizados para os dados de peso-idade:

Brody (B): $P_{i}=a-b e^{-k t_{i}}$

Gompertz (G): $P_{i}=a e^{-b e^{-k t_{i}}}$

Logístico (L): $P_{i}=a\left(1+b e^{-k t_{i}}\right)^{-1}$

Mitscherlich (M) (Batschelet, 1984): 
$P_{i}=a\left(1-e^{-k t_{i}}\right)$

von Bertalanffy (vB): $P_{i}=a\left(1-b e^{-k t_{i}}\right)^{3}$

Richards (R): $P_{i}=a\left(1 \pm b e^{-k t_{i}}\right)^{m}$

Logístico duplo (LD):

$$
P_{i}=\frac{a_{1}}{1+e^{-k\left(t_{i}-b_{1}\right)}}+\frac{a_{2}}{1+e^{-k\left(t_{i}-b_{2}\right)}}
$$

em que $P_{i}$ representa o peso observado no tempo $t_{i}$; $t_{i}$, o tempo decorrido do nascimento à pesagem $i$, em dias; $a$, o peso assintótico; $a_{1}$ e $a_{2}$, os pesos assintóticos na primeira e segunda fases, respectivamente $\left(a_{1}+a_{2}=\mathrm{a}\right) ; b$, uma constante de integração; $b_{1}$ e $b_{2}$, as idades no primeiro e segundo pontos de inflexão, respectivamente; $k$, o índice de maturidade e $m$, o parâmetro de inflexão.

O coeficiente de ajuste $\left(\mathrm{R}^{2}\right)$ para as equações foi calculado como $\mathrm{R}^{2}=1-($ sqe)/(sqt), em que sqe é a soma de quadrados dos erros e sqt, a soma de quadrados total (Kvålseth, 1985). As estimativas obtidas para os parâmetros das equações que convergiram e foram julgadas nos limites considerados biologicamente aceitáveis, ou seja, $a \leq 1000, k \leq 1$ e $a_{1}+a_{2} \leq 1000$, foram analisadas segundo o modelo misto:

$$
\begin{aligned}
& Y_{\text {ijklmnop }}=S_{j}+P_{k}+A_{l}+M_{m}+R_{n}+S_{j} * R_{n}+ \\
& \operatorname{Rep}_{o}+F_{p}+e_{i j k l m n o p}
\end{aligned}
$$

em que: $Y_{\text {ijklmnop }}$ representa a i-ésima observação para a variável $Y, Y=a, a_{1}, a_{2}, a_{1}+a_{2}, b, b_{1}, b_{2}, k, k^{-1}, m$; $S_{j}$, o sexo: $j=1$ (macho), 2 (fêmea); $P_{k}$, a propriedade de criação $(k=7, \ldots, 41) ; A_{l}$, o ano de nascimento ( $l=1960, \ldots, 2000) ; M_{m}$, o mês de nascimento $(m=1$, $\ldots, 12) ; R_{n}$, o tipo de sistema de criação $n=$ a pasto (1), semi-estabulado (2), estabulado (3), com mudança no sistema (4); $S_{j}{ }^{*} R_{n}$, a interação entre o $j$-ésimo sexo e o $n$-ésimo sistema de criação; $\operatorname{Rep}_{o}$, o efeito aleatório do $o$-ésimo reprodutor; $F_{p}$, o efeito aleatório da $p$-ésima mãe; e $e_{i j k l m n o p}$ o erro aleatório associado à i-ésima observação.

Empregou-se o PROC NLIN (SAS, 2000) nas análises dos modelos não-lineares, de modo que as estimativas iniciais empregadas foram obtidas na literatura ou a partir da média dos dados da amostra para todos os parâmetros, quando não disponíveis. Escolheu-se o método de estimação de Gauss-Newton para calcular as estimativas dos parâmetros porque mais simples que o de Newton, mesmo necessitando de um maior número de iterações (Hoffman \& Vieira, 1998). O modelo misto foi analisado utilizando-se o
PROC MIXED (SAS, 2000) com estimativas de máxima verossimilhança restrita, com estrutura de variâncias não-relacionadas (VC). Optou-se pela significância de efeitos fixos e aleatórios até 10\% $(\mathrm{P}<0,10)$, por considerar-se que a variabilidade das estimativas dos parâmetros fosse alta. O critério de informação de Akaike (AIC) foi calculado como $A I C=l(\hat{\theta})-q$, em que $\hat{\theta}$ é a maximização do logaritmo da verossimilhança e $q$, o número de parâmetros de covariância.

\section{Resultados e Discussão}

A escolha dos animais com todas as pesagens do nascimento aos dois anos de idade reduziu consideravelmente o número de observações analisáveis (Figura 1), verificando-se decréscimo de 24.028 para 1.358 no total de animais na amostra. Este comportamento pode ser creditado, em parte, à movimentação inerente à comercialização dos animais e aos eventuais problemas, como mortes, ou ao desinteresse do produtor em continuar com o controle de desenvolvimento ponderal no rebanho.

A presença de maior número de fêmeas que machos provavelmente resultou dos sistemas de manejo dos animais, nos quais as fêmeas seriam abatidas ou comercializadas mais tardiamente, possibilitando seu melhor acompanhamento.

Houve grande variação nos períodos entre pesagens nos grupos não-selecionado e selecionado, embora as estatísticas tenham melhorado após a seleção (Tabela 1). Isso impossibilitou o uso das observações para estimação de uma curva média de crescimento baseada na média dos pesos observados em tempos precisos, embora uma curva média possa ser obtida a partir da média dos parâmetros estimados (Tabelas 2 a 8). A maior variação e a menor média ocorreram para o período entre a primeira (ao nascimento) e a segunda pesagens, para ambos os grupos, o que pode ser explicado pelas dificuldades de manejo impostas a pesagens em idades do nascimento à desmama, em razão da necessidade de separação de mãe e filho, que não ocorre em idades posteriores.

As estimativas para os parâmetros das equações para os animais selecionados (Tabelas 2 a 8) corroboram a bibliografia consultada (Ludwig et al., 1981; Nobre et al., 1987; Perotto et al., 1992; Oliveira et al., 2000).

O número de animais que apresentaram convergência em relação ao total de animais analisados (Tabela 9), 
Tabela 1 - Estatísticas para número de dias entre pesagens para a amostra antes e após a seleção Table 1 - Statistics for number of days between measures before and after selection

\begin{tabular}{|c|c|c|c|c|c|c|c|c|}
\hline \multirow[b]{2}{*}{$\begin{array}{l}\text { Período entre pesagens } \\
\text { Time between measures }\end{array}$} & \multirow[b]{2}{*}{$\begin{array}{c}\text { Estatística } \\
\text { Statistic }\end{array}$} & \multirow[b]{2}{*}{$\begin{array}{l}\text { Amostra } \\
\text { Sample }\end{array}$} & \multicolumn{2}{|c|}{$\begin{array}{l}\text { Guzerá } \\
\text { Guzera }\end{array}$} & \multicolumn{2}{|c|}{$\begin{array}{l}\text { Nelore } \\
\text { Nellore }\end{array}$} & \multicolumn{2}{|c|}{$\begin{array}{l}\text { Nelore Mocho } \\
\text { Polled Nellore }\end{array}$} \\
\hline & & & $\begin{array}{l}\text { Fêmea } \\
\text { Female }\end{array}$ & $\begin{array}{l}\text { Macho } \\
\text { Male }\end{array}$ & $\begin{array}{l}\text { Fêmea } \\
\text { Female }\end{array}$ & $\begin{array}{l}\text { Macho } \\
\text { Male }\end{array}$ & $\begin{array}{l}\text { Fêmea } \\
\text { Female }\end{array}$ & $\begin{array}{l}\text { Macho } \\
\text { Male }\end{array}$ \\
\hline $1-2$ & $\begin{array}{l}\bar{X}^{\mathrm{c}} \\
\mathrm{s}^{2} \\
\mathrm{CV}\end{array}$ & $\begin{array}{l}\mathrm{T} \\
\mathrm{S} \\
\mathrm{T} \\
\mathrm{S} \\
\mathrm{T} \\
\mathrm{S}\end{array}$ & $\begin{array}{r}64,99 \\
53,55 \\
1197,20 \\
767,05 \\
53,24 \\
51,72\end{array}$ & $\begin{array}{r}64,39 \\
51,56 \\
1292,17 \\
683,07 \\
55,82 \\
50,69\end{array}$ & $\begin{array}{r}62,38 \\
48,15 \\
1326,70 \\
852,52 \\
58,39 \\
60,64\end{array}$ & $\begin{array}{r}63,08 \\
46,88 \\
1213,06 \\
829,91 \\
55,22 \\
61,45\end{array}$ & $\begin{array}{r}63,12 \\
53,87 \\
1008,64 \\
748,69 \\
50,31 \\
50,79\end{array}$ & $\begin{array}{r}63,02 \\
46,33 \\
1065,74 \\
830,08 \\
51,80 \\
62,18\end{array}$ \\
\hline $2-3$ & $\begin{array}{l}\bar{x} \\
\mathrm{~s}^{2} \\
\mathrm{CV}\end{array}$ & $\begin{array}{l}\mathrm{T} \\
\mathrm{S} \\
\mathrm{T} \\
\mathrm{S} \\
\mathrm{T} \\
\mathrm{S}\end{array}$ & $\begin{array}{r}92,87 \\
90,98 \\
409,91 \\
116,29 \\
21,80 \\
11,85\end{array}$ & $\begin{array}{r}91,67 \\
92,27 \\
245,14 \\
66,89 \\
17,08 \\
8,86\end{array}$ & $\begin{array}{r}91,82 \\
90,33 \\
207,76 \\
139,93 \\
15,70 \\
13,09\end{array}$ & $\begin{array}{c}91,71 \\
89,06 \\
170,11 \\
12841 \\
14,22 \\
12,72\end{array}$ & $\begin{array}{r}94,38 \\
90,90 \\
559,41 \\
100,14 \\
25,06 \\
1,01\end{array}$ & $\begin{array}{r}95,03 \\
86,17 \\
481,06 \\
69,76 \\
23,08 \\
9,69\end{array}$ \\
\hline $3-4$ & $\begin{array}{l}\bar{x} \\
\mathrm{~s}^{2} \\
\mathrm{CV}\end{array}$ & $\begin{array}{l}\mathrm{T} \\
\mathrm{S} \\
\mathrm{T} \\
\mathrm{S} \\
\mathrm{T} \\
\mathrm{S}\end{array}$ & $\begin{array}{r}90,52 \\
89,90 \\
178,46 \\
116,50 \\
14,76 \\
12,01\end{array}$ & $\begin{array}{r}90,38 \\
88,21 \\
151,06 \\
145,22 \\
13,60 \\
13,66\end{array}$ & $\begin{array}{r}91,95 \\
90,72 \\
211,92 \\
151,69 \\
15,83 \\
13,58\end{array}$ & $\begin{array}{r}91,34 \\
92,05 \\
168,43 \\
123,98 \\
14,21 \\
12,10\end{array}$ & $\begin{array}{r}92,22 \\
93,01 \\
118,39 \\
114,08 \\
11,80 \\
11,48\end{array}$ & $\begin{array}{r}92,58 \\
98,55 \\
109,45 \\
86,05 \\
11,30 \\
9,41\end{array}$ \\
\hline $4-5$ & $\begin{array}{l}\bar{x} \\
\mathrm{~s}^{2} \\
\mathrm{CV}\end{array}$ & $\begin{array}{l}\mathrm{T} \\
\mathrm{S} \\
\mathrm{T} \\
\mathrm{S} \\
\mathrm{T} \\
\mathrm{S}\end{array}$ & $\begin{array}{r}92,53 \\
91,03 \\
249,43 \\
142,28 \\
17,07 \\
13,10\end{array}$ & $\begin{array}{r}92,43 \\
89,69 \\
229,29 \\
215,66 \\
16,38 \\
16,37\end{array}$ & $\begin{array}{r}91,41 \\
91,70 \\
184,91 \\
136,79 \\
14,88 \\
12,75\end{array}$ & $\begin{array}{r}91,45 \\
89,71 \\
152,14 \\
125,73 \\
13,49 \\
12,50\end{array}$ & $\begin{array}{r}91,42 \\
92,17 \\
122,96 \\
118,09 \\
12,13 \\
11,79\end{array}$ & $\begin{array}{r}91,19 \\
85,71 \\
122,40 \\
121,62 \\
12,13 \\
12,87\end{array}$ \\
\hline $5-6$ & $\begin{array}{l}\bar{x} \\
\mathrm{~s}^{2} \\
\mathrm{CV}\end{array}$ & $\begin{array}{l}\mathrm{T} \\
\mathrm{S} \\
\mathrm{T} \\
\mathrm{S} \\
\mathrm{T} \\
\mathrm{S}\end{array}$ & $\begin{array}{r}92,54 \\
94,06 \\
325,85 \\
135,55 \\
19,51 \\
12,38\end{array}$ & $\begin{array}{r}92,55 \\
94,03 \\
136,76 \\
214,36 \\
12,64 \\
15,57\end{array}$ & $\begin{array}{r}92,73 \\
92,00 \\
247,59 \\
135,34 \\
16,97 \\
12,64\end{array}$ & $\begin{array}{r}92,28 \\
93,93 \\
226,00 \\
100,05 \\
16,29 \\
10,65\end{array}$ & $\begin{array}{r}92,53 \\
91,76 \\
157,39 \\
129,71 \\
13,56 \\
12,41\end{array}$ & $\begin{array}{r}93,45 \\
95,94 \\
174,61 \\
141,53 \\
14,14 \\
12,40\end{array}$ \\
\hline $6-7$ & $\begin{array}{l}\bar{x} \\
\mathrm{~s}^{2} \\
\mathrm{CV}\end{array}$ & $\begin{array}{l}\mathrm{T} \\
\mathrm{S} \\
\mathrm{T} \\
\mathrm{S} \\
\mathrm{T} \\
\mathrm{S}\end{array}$ & $\begin{array}{r}92,70 \\
91,76 \\
177,40 \\
111,32 \\
14,37 \\
11,50\end{array}$ & $\begin{array}{r}91,14 \\
91,42 \\
127,28 \\
119,36 \\
12,38 \\
11,95\end{array}$ & $\begin{array}{r}91,45 \\
90,78 \\
150,35 \\
130,26 \\
13,41 \\
12,57\end{array}$ & $\begin{array}{r}91,29 \\
89,63 \\
129,15 \\
96,85 \\
12,45 \\
10,98\end{array}$ & $\begin{array}{l}93,11 \\
92,73 \\
90,16 \\
92,22 \\
10,20 \\
10,36\end{array}$ & $\begin{array}{r}93,98 \\
93,28 \\
90,47 \\
38,56 \\
10,12 \\
6,66\end{array}$ \\
\hline $7-8$ & $\begin{array}{l}\bar{x} \\
\mathrm{~s}^{2} \\
\mathrm{CV}\end{array}$ & $\begin{array}{l}\mathrm{T} \\
\mathrm{S} \\
\mathrm{T} \\
\mathrm{S} \\
\mathrm{T} \\
\mathrm{S}\end{array}$ & $\begin{array}{r}90,59 \\
91,64 \\
158,33 \\
154,40 \\
13,89 \\
13,56\end{array}$ & $\begin{array}{r}91,52 \\
92,56 \\
185,72 \\
160,48 \\
14,89 \\
13,69\end{array}$ & $\begin{array}{r}90,85 \\
91,25 \\
130,52 \\
122,03 \\
12,58 \\
12,11\end{array}$ & $\begin{array}{r}91,18 \\
90,49 \\
123,37 \\
90,43 \\
12,18 \\
10,51\end{array}$ & $\begin{array}{r}92,03 \\
90,67 \\
93,93 \\
117,57 \\
10,53 \\
11,96\end{array}$ & $\begin{array}{r}92,30 \\
85,25 \\
78,59 \\
75,48 \\
9,60 \\
10,19\end{array}$ \\
\hline $8-9$ & $\begin{array}{l}\bar{x} \\
\mathrm{~s}^{2} \\
\mathrm{CV}\end{array}$ & $\begin{array}{l}\mathrm{T} \\
\mathrm{S} \\
\mathrm{T} \\
\mathrm{S} \\
\mathrm{T} \\
\mathrm{S}\end{array}$ & $\begin{array}{r}90,75 \\
91,76 \\
128,10 \\
147,00 \\
12,47 \\
13,21\end{array}$ & $\begin{array}{r}89,75 \\
90,02 \\
159,19 \\
80,44 \\
14,06 \\
9,96\end{array}$ & $\begin{array}{r}92,75 \\
92,60 \\
202,27 \\
145,50 \\
15,33 \\
13,03\end{array}$ & $\begin{array}{r}92,88 \\
92,32 \\
219,36 \\
116,58 \\
15,95 \\
11,70\end{array}$ & $\begin{array}{r}91,02 \\
88,80 \\
133,97 \\
185,34 \\
12,72 \\
15,33\end{array}$ & $\begin{array}{r}95,76 \\
92,94 \\
85,18 \\
69,20 \\
9,64 \\
8,95\end{array}$ \\
\hline
\end{tabular}

c $\bar{X}=$ média; $\mathrm{s}^{2}=$ variância; $\mathrm{CV}=$ coeficiente de variação; $\mathrm{T}=$ antes da seleção; $\mathrm{S}=$ após a seleção.

$c \bar{X}=$ average; $s^{2}=$ variance; $C V=$ coefficient of variation; $T=$ before selection; $S=$ after selection. 


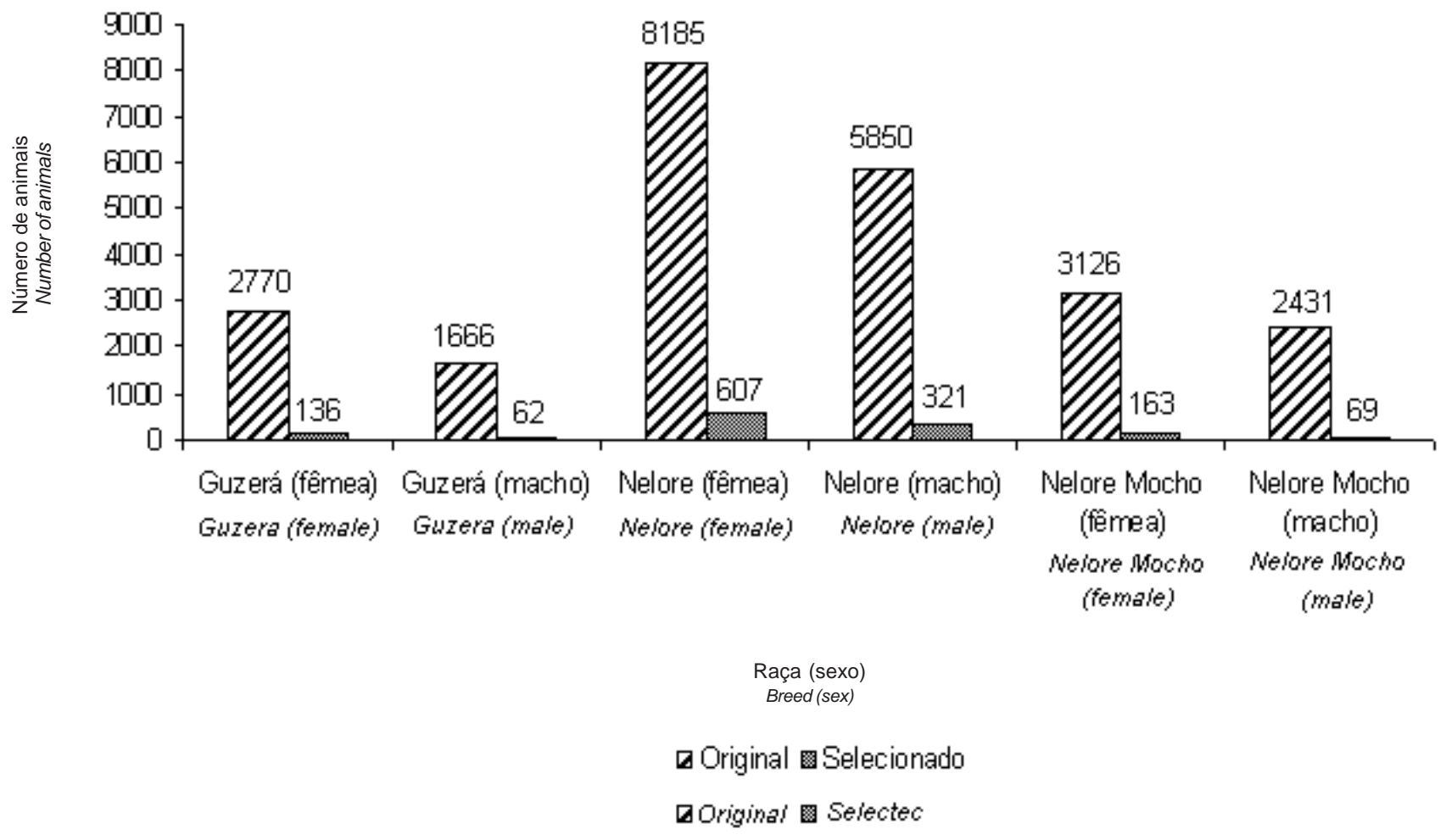

Figura 1 - Total de animais na amostra antes e após a seleção dos dados.

Figure 1 - Total number of animals in the sample before and after record selection.

juntamente com os coeficientes de ajuste (Tabelas 2 a 8 e 10), forneceu informações para se considerar o modelo logístico duplo o mais adequado para todas as raças e sexos sob o ponto de vista de facilidade computacional, enquanto os modelos de Richards e Brody foram superiores quanto à interpretação biológica de seus parâmetros. Outros autores consideraram melhores os modelos Brody (Nobre et al., 1987; Ludwig et al., 1981), Richards (Nobre et al., 1987; Goonewardene et al., 1981), von Bertalanffy (Oliveira et al., 2000) e logístico duplo (Koenen \& Groen, 1996).

O segundo melhor modelo, analisando-se somente o coeficiente de ajuste, foi o de Richards (Tabela 10), mas mostrou-se pouco satisfatório por apresentar o menor número de convergências (Tabela 9), o que, segundo Rutledge et al. (1972) e Brown et al. (1976), pode ser atribuído primeiramente à alta correlação entre os parâmetros $k$ e $m$, apesar do coeficiente de ajuste geralmente superior, justificado pela flexibilidade do parâmetro $m$. Segundo Doren et al. (1989), o modelo de Richards teria problemas de ajuste aos dados após o período de inflexão, que seria a transição da puberdade à idade adulta. Isso explicaria, em parte, o pequeno número de convergências obtido para a amostra, pois o ponto de inflexão estaria pouco definido, uma vez que a idade máxima de coleta de dados foi de dois anos e, a partir daí, provavelmente os animais continuaram a se desenvolver, implicando em perda de informações e afetando o cálculo do ponto de inflexão "real", justificando o acompanhamento dos animais até idades superiores a dois anos. Portanto, modelo se adequa melhor a bovinos europeus com observações até dois anos de idade, pois a maturidade desses animais é atingida dentro deste período, o que não ocorre com animais zebuínos.

Os maiores pesos assintóticos foram obtidos, em geral, para a raça Nelore e as menores taxas de maturação, para a raça Guzerá, apesar de nem sempre terem sido significativamente diferente dos pesos das outras raças (Tabela 11). Assim, os animais da raça Guzerá poderiam atingir peso assintótico igual aos das demais raças somente em idade mais avançada, em razão de seu desenvolvimento mais lento, mas poderiam alcançar peso adulto mais elevado que aqueles em idades superiores. A indisponibilidade de dados em idades superiores a dois anos não possibilitou 
Tabela 2 - Medidas descritivas dos parâmetros médios estimados pela equação Brody Table 2 - Brody equation parameters mean estimates

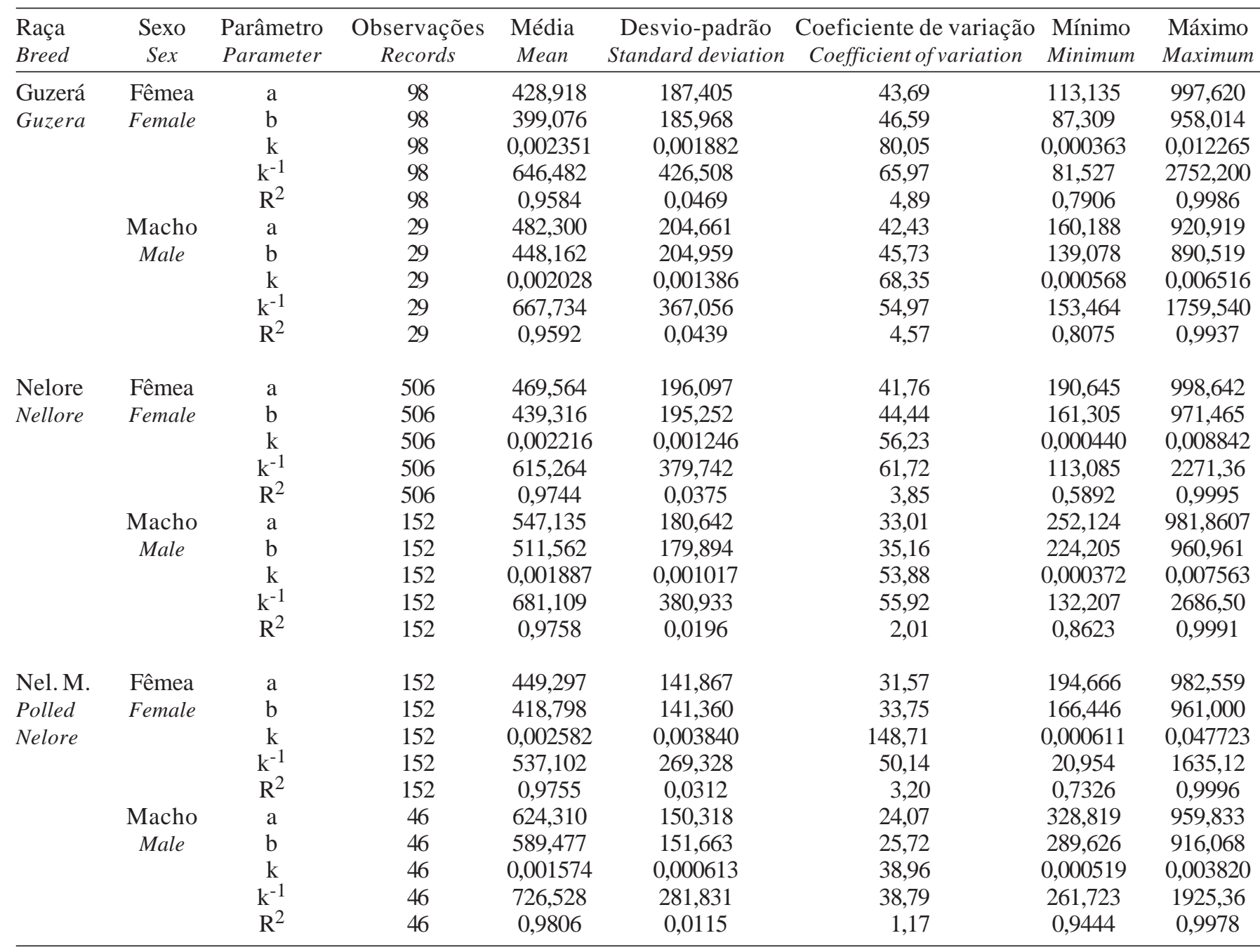

a verificação desse fato. Essa falha no acompanhamento dos animais deveria ser corrigida, o que colaboraria com maior precisão da estimativa da curva de crescimento (Ludwig et al., 1981; Nobre et al., 1987).

As equações de crescimento utilizadas partem de diferentes pressupostos para o seu ajuste aos dados, como evidenciado, por exemplo, pelos diferentes pontos de inflexão considerados pelos modelos. Isto acarretou diferentes estimações para peso assintótico (a ou $\left.\mathrm{a}_{1}+\mathrm{a}_{2}\right)$ e idade à maturidade $\left(\mathrm{k}^{-1}\right)$, entre outros parâmetros. Essas estimativas também apresentaram variações distintas, tanto entre modelos quanto entre 
Tabela 3 - Medidas descritivas dos parâmetros médios estimados pela equação Gompertz Table 3 - Gompertz equation parameters mean estimates

\begin{tabular}{|c|c|c|c|c|c|c|c|c|}
\hline $\begin{array}{l}\text { Raça } \\
\text { Breed }\end{array}$ & $\begin{array}{l}\text { Sexo } \\
\text { Sex }\end{array}$ & $\begin{array}{l}\text { Parâmetro } \\
\text { Parameter }\end{array}$ & $\begin{array}{l}\text { Observações } \\
\text { Records }\end{array}$ & $\begin{array}{l}\text { Média } \\
\text { Mean }\end{array}$ & $\begin{array}{l}\text { Desvio-padrão } \\
\text { Standard deviation }\end{array}$ & $\begin{array}{c}\text { Coeficiente de variação } \\
\text { Coefficient of variation }\end{array}$ & $\begin{array}{l}\text { Mínimo } \\
\text { Minimum }\end{array}$ & $\begin{array}{l}\text { Máximo } \\
\text { Maximum }\end{array}$ \\
\hline Guzerá & Fêmea & a & 129 & 380,755 & 147,756 & 38,80 & 141,112 & 902,865 \\
\hline \multirow[t]{9}{*}{ Guzera } & \multirow[t]{4}{*}{ Female } & $\mathrm{b}$ & 129 & 2,215 & 0,359 & 16,24 & 1,458 & 3,087 \\
\hline & & $\mathrm{k}$ & 129 & 0,004404 & 0,002296 & 52,13 & 0,001054 & 0,017034 \\
\hline & & $\mathrm{k}^{-1}$ & 129 & 281,158 & 139,966 & 49,78 & 58,705 & 948,167 \\
\hline & & $\mathrm{R}^{2}$ & 129 & 0,9638 & 0,0373 & 3,87 & 0,7738 & 0,998510 \\
\hline & Macho & $\mathrm{a}$ & 55 & 463,228 & 175,602 & 37,90 & 139,247 & 926,756 \\
\hline & \multirow[t]{4}{*}{ Male } & b & 55 & 2,298 & 0,407 & 17,71 & 1,521 & 3,180 \\
\hline & & $\mathrm{k}$ & 55 & 0,003707 & 0,001701 & 45,89 & 0,001340 & 0,010579 \\
\hline & & $\mathrm{k}^{-1}$ & 55 & 322,791 & 141,365 & 43,79 & 94,527 & 746,015 \\
\hline & & $\mathrm{R}^{2}$ & 55 & 0,9655 & 0,033267 & 3,44 & 0,8372 & 0,9986 \\
\hline Nelore & Fêmea & $\mathrm{a}$ & 597 & 379,072 & 129,550 & 34,17 & 174,690 & 937,233 \\
\hline \multirow[t]{9}{*}{ Nellore } & \multirow[t]{4}{*}{ Female } & $\mathrm{b}$ & 597 & 2,218 & 0,656 & 29,57 & 1,433 & 16,243 \\
\hline & & $\mathrm{k}$ & 597 & 0,004849 & 0,002094 & 43,19 & 0,001139 & 0,030337 \\
\hline & & $\mathrm{k}^{-1}$ & 597 & 240,644 & 106,486 & 44,25 & 32,962 & 877,944 \\
\hline & & $\mathrm{R}^{2}$ & 597 & 0,9727 & 0,0355 & 3,65 & 0,5926 & 0,9989 \\
\hline & Macho & $\mathrm{a}$ & 298 & 540,091 & 187,936 & 34,79 & 227,567 & 999,445 \\
\hline & \multirow[t]{4}{*}{ Male } & $\mathrm{b}$ & 298 & 2,378 & 0,387 & 16,29 & 1,607 & 3,613 \\
\hline & & $\mathrm{k}$ & 298 & 0,003897 & 0,001457 & 37,38 & 0,001205 & 0,012387 \\
\hline & & $\mathrm{k}^{-1}$ & 298 & 291,474 & 109,504 & 37,56 & 80,729 & 829,684 \\
\hline & & $\mathrm{R}^{2}$ & 298 & 0,9762 & 0,0225 & 2,31 & 0,8687 & 0,9998 \\
\hline Nel. M. & Fêmea & $\mathrm{a}$ & 163 & 365,650 & 93,902 & 25,68 & 182,989 & 779,126 \\
\hline Polled & \multirow[t]{4}{*}{ Female } & $\mathrm{b}$ & 163 & 2,137 & 0,252 & 11,79 & 1,064 & 2,861 \\
\hline \multirow[t]{8}{*}{ Nellore } & & $\mathrm{k}$ & 163 & 0,005065 & 0,001733 & 34,21 & 0,001700 & 0,014959 \\
\hline & & $\mathrm{k}^{-1}$ & 163 & 218,911 & 75,730 & 34,59 & 66,847 & 588,083 \\
\hline & & $\mathrm{R}^{2}$ & 163 & 0,9705 & 0,0321 & 3,31 & 0,7314 & 0,9968 \\
\hline & Macho & $\mathrm{a}$ & 66 & 497,838 & 134,765 & 27,07 & 302,129 & 929,761 \\
\hline & \multirow[t]{4}{*}{ Male } & b & 66 & 2,370 & 0,690 & 29,13 & 1,780 & 7,536 \\
\hline & & $\mathrm{k}$ & 66 & 0,004048 & 0,001153 & 28,50 & 0,001945 & 0,007558 \\
\hline & & $\mathrm{k}^{-1}$ & 66 & 268,651 & 82,855 & 30,84 & 132,305 & 513,955 \\
\hline & & $\mathrm{R}^{2}$ & 66 & 0,9758 & 0,0157 & 1,61 & 0,9143 & 0,9985 \\
\hline
\end{tabular}

raças para um mesmo modelo, implicando diretamente em diferentes estimativas para a influência de efeitos fixos e aleatórios, como demonstrado a seguir.

A qualidade de ajuste do modelo misto encontra-se na Tabela 12, pela estatística de qualidade de ajuste pelo critério de informação de Akaike (AIC) e pela redução em $\sigma_{e}^{2}$. Os resultados evidenciarama possibilidade de exploração da modificação da curva de crescimento por meio de seus parâmetros, pois a variabilidade no rebanho proporcionada pelas progenitoras foi 
Tabela 4 - Medidas descritivas dos parâmetros médios estimados pela equação Logística Table 4 - Logistic equation parameters mean estimates

\begin{tabular}{|c|c|c|c|c|c|c|c|c|}
\hline $\begin{array}{l}\text { Raça } \\
\text { Breed }\end{array}$ & $\begin{array}{l}\text { Sexo } \\
\text { Sex }\end{array}$ & $\begin{array}{l}\text { Parâmetro } \\
\text { Parameter }\end{array}$ & $\begin{array}{c}\text { Observações } \\
\text { Records }\end{array}$ & $\begin{array}{l}\text { Média } \\
\text { Mean }\end{array}$ & $\begin{array}{l}\text { Desvio-padrão } \\
\text { Standard deviation }\end{array}$ & $\begin{array}{c}\text { Coeficiente de variação } \\
\text { Coefficient of variation }\end{array}$ & $\begin{array}{l}\text { Mínimo } \\
\text { Minimum }\end{array}$ & $\begin{array}{l}\text { Máximo } \\
\text { Maximum }\end{array}$ \\
\hline Guzerá & Fêmea & a & 83 & 352,216 & 111,7918 & 31,73 & 111,930 & 631,354 \\
\hline \multirow[t]{9}{*}{ Guzera } & Female & b & 83 & 6,382 & 1,967 & 30,83 & 2,757 & 11,241 \\
\hline & & $\mathrm{k}$ & 83 & 0,007660 & 0,003773 & 49,25 & 0,003012 & 0,023874 \\
\hline & & $\mathrm{k}^{-1}$ & 83 & 151,818 & 53,446 & 35,20 & 41,885 & 331,968 \\
\hline & & $\mathrm{R}^{2}$ & 83 & 0,9609 & 0,0415 & 4,32 & 0,7744 & 0,9982 \\
\hline & Macho & $\mathrm{a}$ & 46 & 489,999 & 186,540 & 38,06 & 205,998 & 972,250 \\
\hline & Male & b & 46 & 7,646 & 2,905 & 38,00 & 3,309 & 16,195 \\
\hline & & $\mathrm{k}$ & 46 & 0,005966 & 0,001809 & 30,33 & 0,003177 & 0,012760 \\
\hline & & $\mathrm{k}^{-1}$ & 46 & 182,348 & 53,599 & 29,39 & 78,364 & 314,671 \\
\hline & & $\mathrm{R}^{2}$ & 46 & 0,9708 & 0,0221 & 2,27 & 0,8925 & 0,998121 \\
\hline Nelore & Fêmea & $\mathrm{a}$ & 378 & 374,820 & 113,952 & 30,40 & 168,047 & 836,192 \\
\hline \multirow[t]{9}{*}{ Nellore } & Female & b & 378 & 6,322 & 1,875 & 29,66 & 2,820 & 16,096 \\
\hline & & $\mathrm{k}$ & 378 & 0,007854 & 0,002523 & 32,13 & 0,002659 & 0,020209 \\
\hline & & $\mathrm{k}^{-1}$ & 378 & 140,924 & 48,590 & 34,47 & 49,481 & 376,031 \\
\hline & & $\mathrm{R}^{2}$ & 378 & 0,9692 & 0,0308 & 3,17 & 0,6828 & 0,9977 \\
\hline & Macho & $\mathrm{a}$ & 256 & 503,297 & 138,811 & 27,58 & 251,280 & 954,448 \\
\hline & Male & b & 256 & 7,452 & 2,443 & 32,79 & 3,2990 & 19,602 \\
\hline & & $\mathrm{k}$ & 256 & 0,006495 & 0,001973 & 30,37 & 0,002578 & 0,017449 \\
\hline & & $\mathrm{k}^{-1}$ & 256 & 167,063 & 48,257 & 28,88 & 57,308 & 387,819 \\
\hline & & $\mathrm{R}^{2}$ & 256 & 0,9717 & 0,0252 & 2,59 & 0,8699 & 0,9986 \\
\hline Nel. M. & Fêmea & a & 123 & 349,103 & 73,672 & 21,10 & 177,996 & 654,853 \\
\hline Polled & Female & b & 123 & 5,808 & 1,348 & 23,22 & 2,875 & 9,847 \\
\hline \multirow[t]{8}{*}{ Nellore } & & $\mathrm{k}$ & 123 & 0,009308 & 0,012670 & 136,11 & 0,003462 & 0,145337 \\
\hline & & $\mathrm{k}^{-1}$ & 123 & 131,457 & 36,912 & 28,07 & 6,880 & 288,853 \\
\hline & & $\mathrm{R}^{2}$ & 123 & 0,9646 & 0,0348 & 3,60 & 0,7332 & 0,9950 \\
\hline & Macho & $\mathrm{a}$ & 61 & 458,541 & 119,280 & 26,01 & 292,207 & 836,273 \\
\hline & Male & b & 61 & 7,393 & 7,132 & 96,47 & 3,977 & 61,021 \\
\hline & & $\mathrm{k}$ & 61 & 0,006744 & 0,001744 & 25,86 & 0,004112 & 0,013071 \\
\hline & & $\mathrm{k}^{-1}$ & 61 & 157,622 & 38,975 & 24,72 & 76,503 & 243,145 \\
\hline & & $\mathrm{R}^{2}$ & 61 & 0,9685 & 0,0167 & 1,731 & 0,9273 & 0,9942 \\
\hline
\end{tabular}

significativa na maioria dos casos, enquanto, para os reprodutores, foi significativa apenas em alguns casos.

O peso assintótico foi afetado pelos efeitos de reprodutor e fêmea: $\sigma_{\text {Rep }}^{2}$ e $\sigma_{\mathrm{F}}^{2}$, respectivamente, para as equações Mitscherlich e von Bertalanffy, para a raça Nelore, e Mitscherlich, para a raça Nelore Mocho. Programas de melhoramento genético pode- riam usufruir dessa variabilidade por meio do acasalamento de animais com valores superiores, alterando o tempo necessário para atingir a maturidade $\left(\mathrm{k}^{-1}\right)$, de modo que o peso máximo seria atingido em idades inferiores.

A taxa de maturidade da raça Nelore Mocho foi influenciada em todas as equações pela fêmea e, pelo 
Tabela 5 - Medidas descritivas dos parâmetros médios estimados pela equação Mitscherlich Table 5 - Mitscherlich equation parameters mean estimates

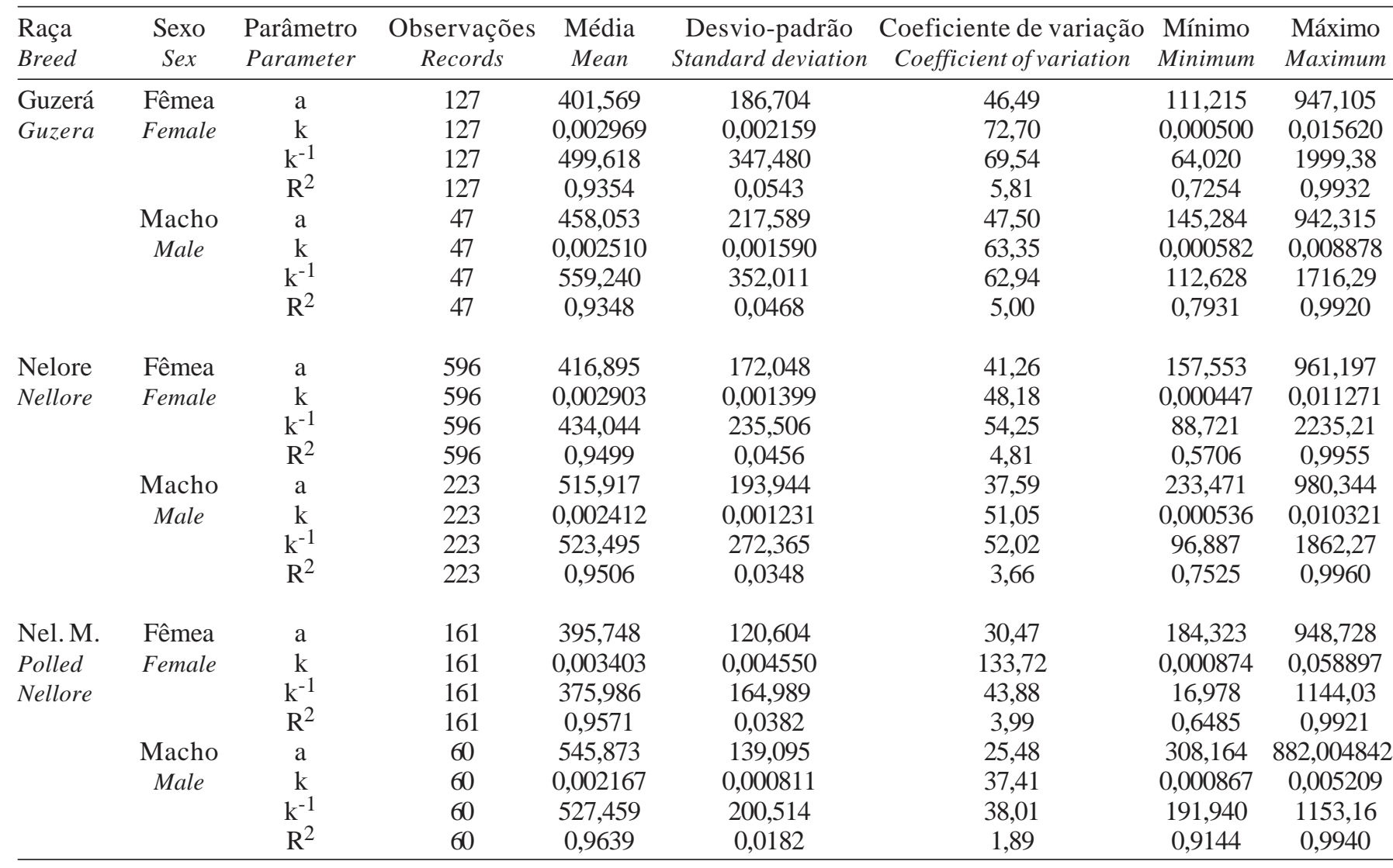

reprodutor, apenas na Mitscherlich. A variabilidade apontou para a possibilidade de alteração no grau de maturidade dos descendentes a partir da correta seleção dos pais.

De modo geral, a fêmea teve maior influência que o macho, evidenciando a importância do correto ma- nejo desses animais no rebanho, seja sob o aspecto nutricional, em que a correta alimentação propiciaria melhor desenvolvimento ao descendente por meio do fornecimento do leite, diminuindo o tempo necessário para atingir a maturidade, seja do ponto de vista do melhoramento, em que o fator genético materno teve 
Tabela 6 - Medidas descritivas dos parâmetros médios estimados pela equação von Bertalanffy Table 6 - von Bertalanffy equation parameters mean estimates

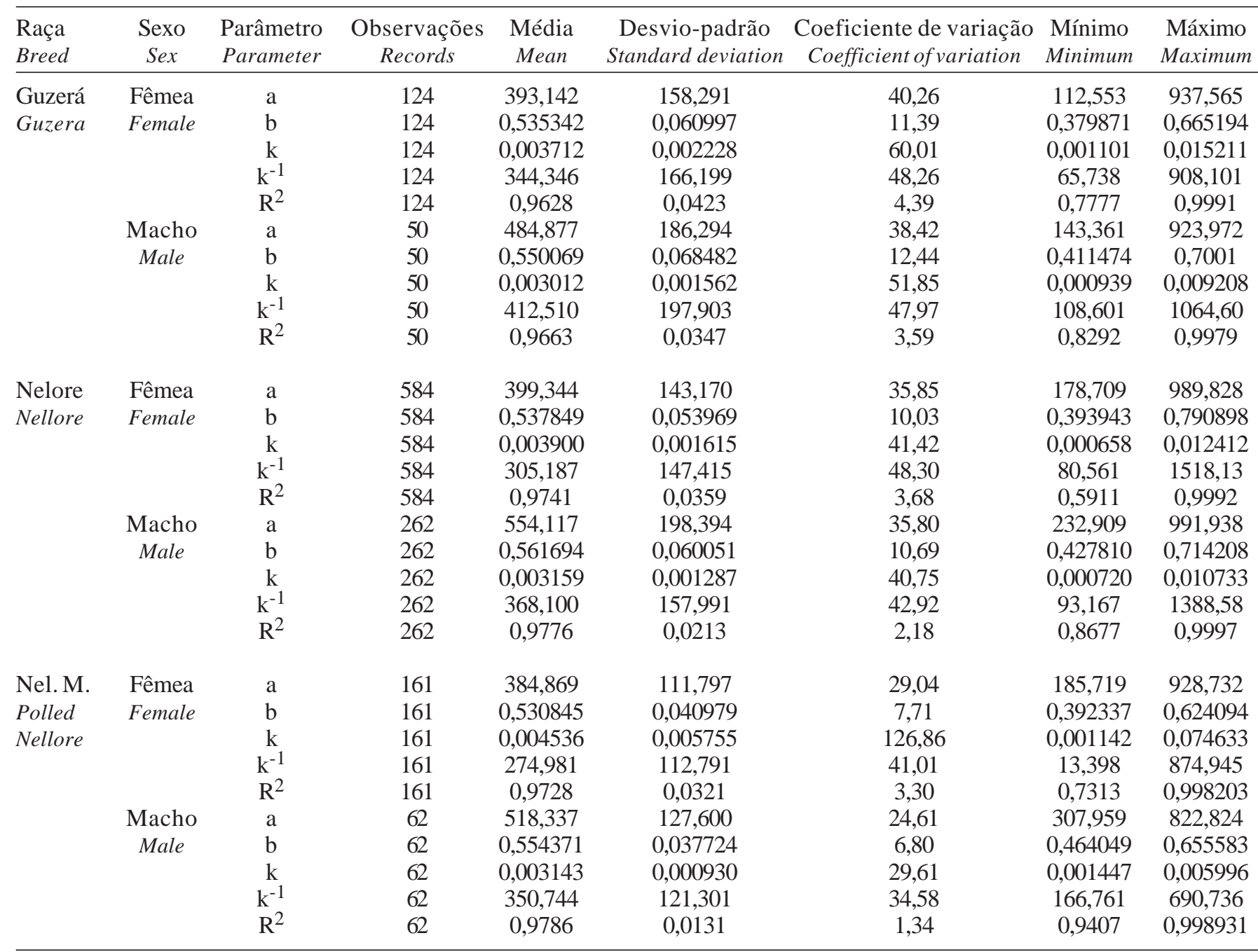

maior variabilidade que o paterno. Portanto, conforme a amostra estudada, maior atenção deve ser dada às fêmeas em um possível programa de melhoramento genético.

A idéia de mudança no formato da curva de crescimento foi sugerida por Tallis (1968), que traba- lhou a construção de um índice de seleção $I$ que modificasse uma curva pré-existente em alguns ou em todos os pontos. Fitzhugh Jr. (1976) analisou vários aspectos da utilização de modelos da família de Richards, discutindo métodos para alteração do for- 
Tabela 7 - Medidas descritivas dos parâmetros médios estimados pela equação Richards Table 7 - Richards equation parameters mean estimates

\begin{tabular}{|c|c|c|c|c|c|c|c|c|}
\hline $\begin{array}{l}\text { Raça } \\
\text { Breed }\end{array}$ & $\begin{array}{l}\text { Sexo } \\
\text { Sex }\end{array}$ & $\begin{array}{l}\text { Parâmetro } \\
\text { Parameter }\end{array}$ & $\begin{array}{c}\text { Observações } \\
\text { Records }\end{array}$ & $\begin{array}{l}\text { Média } \\
\text { Mean }\end{array}$ & $\begin{array}{l}\text { Desvio-padrão } \\
\text { Standard deviation }\end{array}$ & $\begin{array}{c}\text { Coeficiente de variação } \\
\text { Coefficient of variation }\end{array}$ & $\begin{array}{l}\text { Mínimo } \\
\text { Minimum }\end{array}$ & $\begin{array}{l}\text { Máximo } \\
\text { Maximum }\end{array}$ \\
\hline Guzerá & Fêmea & $\mathrm{a}$ & 30 & 475,838 & 214,670 & 45,11 & 214,284 & 991,109 \\
\hline \multirow{8}{*}{ Guzera } & & $\mathrm{k}$ & 30 & 0,002404 & 0,001216 & 50,59 & 0,000092 & 0,005090 \\
\hline & & $\mathrm{k}^{-1}$ & 30 & 871,053 & 1911,82 & 219,48 & 196,444 & 10838,77 \\
\hline & & $\mathrm{m}$ & 30 & 1,284192 & 0,636412 & 49,55 & 0,553303 & 3,096886 \\
\hline & Male & b & 7 & 0,651698 & 0,190619 & 29,24 & 0,342966 & 0,843298 \\
\hline & & $\mathrm{k}$ & 7 & 0,002687 & 0,001180 & 43,91 & 0,000798 & 0,004293 \\
\hline & & $\mathrm{m}$ & 7 & 2,939347 & 1,462005 & 49,73 & 1,713397 & 5,669456 \\
\hline & & $\mathrm{k}^{-1}$ & 7 & 492,058 & 356,921 & 72,53 & 232,893 & 1252,94 \\
\hline & & $\mathrm{R}^{2}$ & 7 & 0,9935 & 0,0041 & 0,41 & 0,9858 & 0,9980 \\
\hline \multirow{9}{*}{ Nellore } & & $\mathrm{k}^{-1}$ & 222 & 608,702 & 533,927 & 87,71 & 149,471 & 3807,84 \\
\hline & & $\mathrm{m}$ & 222 & 1,519427 & 1,914213 & 125,98 & 0,585001 & 24,475432 \\
\hline & & $\mathrm{R}^{2}$ & 222 & 0,9857 & 0,0200 & 2,03 & 0,7904 & 0,9996 \\
\hline & Macho & $\mathrm{a}$ & 58 & 629,900 & 196,507 & 31,19 & 313,491 & 990,063 \\
\hline & Male & b & 58 & 0,883492 & 0,133933 & 15,15 & 0,423529 & 0,995094 \\
\hline & & $\mathrm{k}$ & 58 & 0,001817 & 0,000945 & 52,04 & 0,000311 & 0,005215 \\
\hline & & $\mathrm{k}^{-1}$ & 58 & 762,408 & 543,414 & 71,27 & 191,725 & 3214,38 \\
\hline & & $\mathrm{m}$ & 58 & 1,379242 & 0,902989 & 65,46 & 0,5634127 & 4,826415 \\
\hline & & $\mathrm{R}^{2}$ & 58 & 0,9883 & 0,0107 & 1,08 & 0,9568 & 0,9993 \\
\hline Nel. M. & Fêmea & $\mathrm{a}$ & 89 & 449,965 & 147,314 & 32,73 & 205,459 & 955,722 \\
\hline Polled & Female & b & 89 & 0,868034 & 0,147569 & 17,00 & 0,130778 & 0,996751 \\
\hline \multirow{3}{*}{ Nellore } & & $\mathrm{k}^{-1}$ & 22 & 647,386 & 386,705 & 59,73 & 264,787 & 1441,16 \\
\hline & & $\mathrm{m}$ & 22 & 1,478789 & 0,996515 & 67,38 & 0,593461 & 4,297190 \\
\hline & & $\mathrm{R}^{2}$ & 22 & 0,9869 & 0,0136 & 1,38 & 0,9445 & 0,9990 \\
\hline
\end{tabular}


Tabela 8 - Medidas descritivas dos parâmetros médios estimados pela equação Logístico Duplo Table 8 - Double logistic equation parameters mean estimates

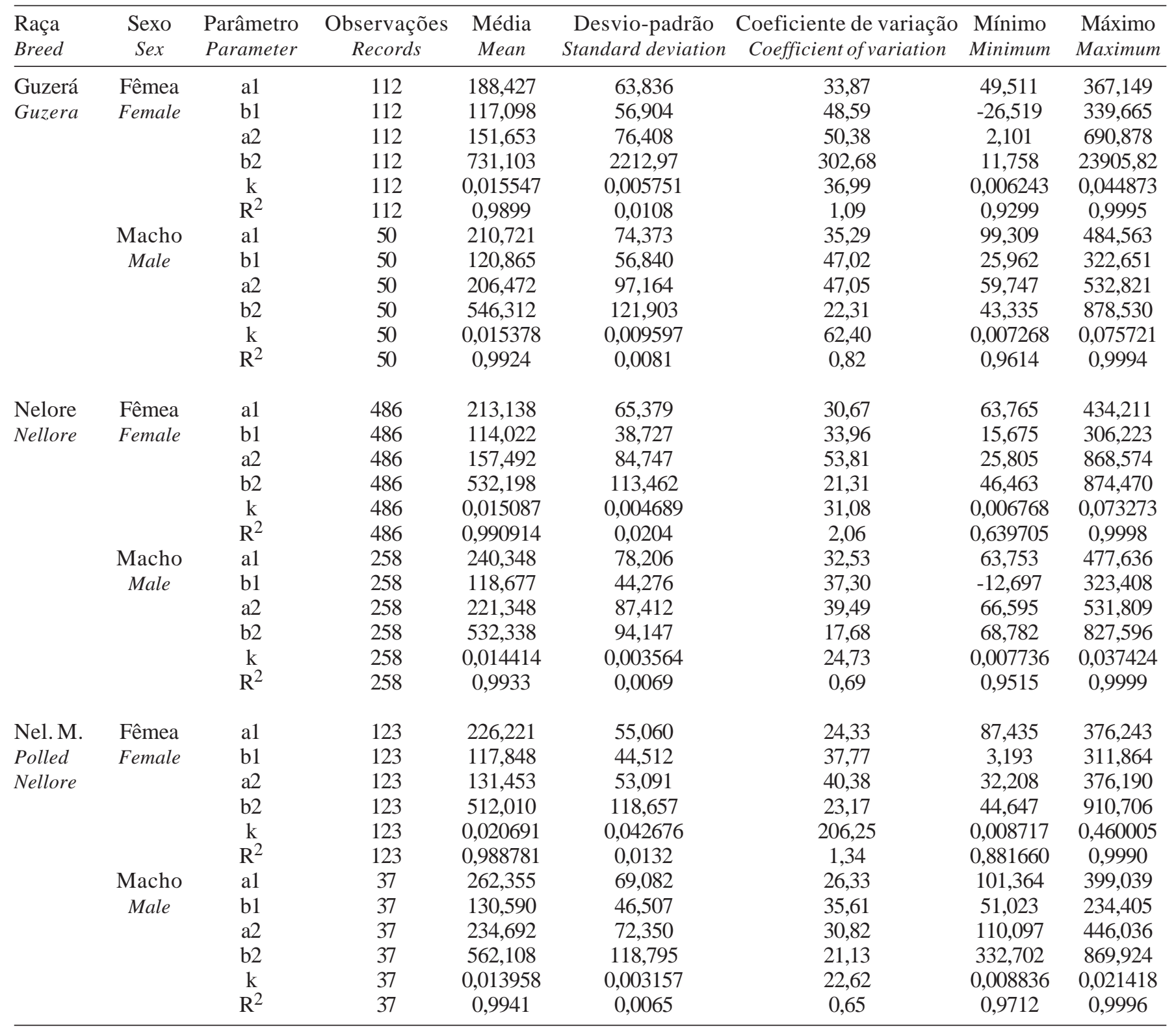


Tabela 9 - Número de animais para os quais as equações convergiram em relação ao total de animais analisados e representatividade, em porcentagem

Table 9 - Number of animals for which the equation had obtained convergence in relation with the total analyzed, \%

\begin{tabular}{|c|c|c|c|c|c|c|}
\hline & \multicolumn{2}{|c|}{$\begin{array}{l}\text { Guzerá } \\
\text { Guzera }\end{array}$} & \multicolumn{2}{|c|}{$\begin{array}{l}\text { Nelore } \\
\text { Nellore }\end{array}$} & \multicolumn{2}{|c|}{$\begin{array}{l}\text { Nelore Mocho } \\
\text { Polled Nellore }\end{array}$} \\
\hline & $\begin{array}{l}\text { Fêmea } \\
\text { Female }\end{array}$ & $\begin{array}{l}\text { Macho } \\
\text { Male }\end{array}$ & $\begin{array}{l}\text { Fêmea } \\
\text { Female }\end{array}$ & $\begin{array}{l}\text { Macho } \\
\text { Male }\end{array}$ & $\begin{array}{l}\text { Fêmea } \\
\text { Female }\end{array}$ & $\begin{array}{l}\text { Macho } \\
\text { Male }\end{array}$ \\
\hline $\begin{array}{l}\text { Modelo } \\
\text { Model }\end{array}$ & $136^{\mathrm{a}}$ & 62 & 607 & 321 & 163 & 69 \\
\hline $\begin{array}{l}\text { Brody } \\
\text { Brody }\end{array}$ & $98(72,06 \%)$ & $29(46,77 \%)$ & $506(83,36 \%)$ & $152(47,35 \%)$ & $152(93,25 \%)$ & 46(66,67\%) \\
\hline $\begin{array}{l}\text { Gompertz } \\
\text { Gompertz }\end{array}$ & $129(94,85 \%)$ & $55(88,71 \%)$ & $597(98,35 \%)$ & 298(92,83\%) & $163(100,00 \%)$ & $66(95,65 \%)$ \\
\hline $\begin{array}{l}\text { Logístico } \\
\text { Logistic }\end{array}$ & $83(61,03 \%)$ & $46(74,19 \%)$ & $378(62,27 \%)$ & $256(79,75 \%)$ & $123(75,46 \%)$ & $61(88,40 \%)$ \\
\hline $\begin{array}{l}\text { Mitscherlich } \\
\text { Mitscherlich }\end{array}$ & $127(93,38 \%)$ & $47(75,80 \%)$ & $596(98,18 \%)$ & $223(69,47 \%)$ & $181(98,77 \%)$ & $60(86,95 \%)$ \\
\hline $\begin{array}{l}\text { von Bertalanffy } \\
\text { Von Bertalanffy }\end{array}$ & $124(91,17 \%)$ & $50(80,64 \%)$ & $584(96,21 \%)$ & $262(81,62 \%)$ & 161(98,77\%) & $62(89,85 \%)$ \\
\hline $\begin{array}{l}\text { Richards } \\
\text { Richards }\end{array}$ & $30(22,06 \%)$ & $7(11,29 \%)$ & $222(36,57 \%)$ & $58(18,07 \%)$ & $89(54,60 \%)$ & $22(31,88 \%)$ \\
\hline $\begin{array}{l}\text { Logístico duplo } \\
\text { Double logistic }\end{array}$ & $112(82,35 \%)$ & $50(80,64 \%)$ & $486(80,06 \%)$ & $258(80,37 \%)$ & $123(75,46 \%)$ & $37(53,62 \%)$ \\
\hline
\end{tabular}

a Número total de animais sob análise.

a Total number of animals under analysis.

Tabela 10 - Teste para a diferença entre médias para 0 coeficiente de ajuste para os diferentes modelos utilizados para todas as raças estudadas

Table 10 - Test for difference between means of adjusted coefficient for different models used for all breeds

\begin{tabular}{|c|c|c|}
\hline $\begin{array}{l}\text { Modelo } \\
\text { Model }\end{array}$ & $\begin{array}{l}\text { Média } \\
\text { Mean }\end{array}$ & Student-Newman-Keuls \\
\hline $\begin{array}{l}\text { Logístico duplo } \\
\text { Double logistic }\end{array}$ & 0,991341 & A \\
\hline $\begin{array}{l}\text { Richards } \\
\text { Richards }\end{array}$ & 0,985321 & B \\
\hline $\begin{array}{l}\text { Von Bertalanffy } \\
\text { Von Bertalanffy }\end{array}$ & 0,973512 & $\mathrm{C}$ \\
\hline $\begin{array}{l}\text { Brody } \\
\text { Brody }\end{array}$ & 0,973071 & $\mathrm{C}$ \\
\hline $\begin{array}{l}\text { Gompertz } \\
\text { Gompertz }\end{array}$ & 0,972235 & $\mathrm{C}$ \\
\hline $\begin{array}{l}\text { Logístico } \\
\text { Logistic }\end{array}$ & 0,968650 & D \\
\hline $\begin{array}{l}\text { Mitscherlich } \\
\text { Mitscherlich }\end{array}$ & 0,949602 & $\mathrm{E}$ \\
\hline
\end{tabular}

* Linhas com letras distintas na mesma coluna diferem a 5\% $(P<0,05)$.

* Rows with different characters on same column differ at $5 \%(P<0.05)$. mato da curva de crescimento e para construção de índices e critérios de seleção.

A raça Hereford foi estimada como $28 \%$ superior em maturação ( $k$ ) aos cruzamentos Charolês, Angus e Galloway (Goonewardene et al., 1981). No Brasil, Rosa et al. (1979), trabalhando com dados da raça Nelore, indicaram o critério de seleção para peso aos 12 meses como o mais adequado na tentativa de se aumentar a precocidade sem aumentar excessivamente o peso adulto, como respostas correlacionadas à seleção. Testando um cruzamento delineado para explorar a heterose total, Perotto et al. (1994) concluíram que o formato da curva de crescimento dos descendentes tinha sido alterado em relação aos animais fundadores do rebanho. Ao analisarem dados de fêmeas Gir, Guzerá, Holandês x Gir e Holandês x Guzerá, Perotto et al. (1997) relataram que o cruzamento Holandês x Zebu aumentou o peso adulto sem diminuir muito a taxa de maturação. Esses resultados implicam que a variabilidade genética ocorre na amostra estudada e que ela pode ser utilizada na mudança da forma da curva de crescimento. sendo que ela ocorre na amostra estudada. 
Tabela 11 - Teste para a diferença entre médias para o peso assintótico (a) e a taxa de maturidade ( $k$ ) entre os diferentes modelos utilizados para todas as raças estudadas

Table 11 - Test for difference between means of asymptotic weight (a) and maturity rate for different models used for all breeds

\begin{tabular}{|c|c|c|c|c|c|}
\hline \multirow[b]{3}{*}{$\begin{array}{l}\text { Modelo } \\
\text { Model }\end{array}$} & \multirow[b]{3}{*}{$\begin{array}{l}\text { Raça } \\
\text { Breed }\end{array}$} & \multicolumn{4}{|c|}{$\begin{array}{l}\text { Parâmetro } \\
\text { Parameter }\end{array}$} \\
\hline & & \multicolumn{2}{|c|}{$\mathrm{a}$} & \multicolumn{2}{|c|}{$\mathrm{k}$} \\
\hline & & $\begin{array}{l}\text { Média } \\
\text { Mean }\end{array}$ & $\begin{array}{c}\text { SNK* } \\
\text { SNK }\end{array}$ & $\begin{array}{l}\text { Média } \\
\text { Mean }\end{array}$ & $\begin{array}{l}\text { SNK } \\
\text { SNK } \\
\end{array}$ \\
\hline $\begin{array}{l}\text { Logístico duplo } \\
\text { Double logistic }\end{array}$ & $\begin{array}{c}\text { Guzerá(Guzera) } \\
\text { Nelore (Nellore) } \\
\text { Nelore Mocho (Polled Nellore) }\end{array}$ & $\begin{array}{l}363,88 \\
402,21 \\
389,90\end{array}$ & $\begin{array}{l}\text { A } \\
\text { B } \\
\text { B }\end{array}$ & $\begin{array}{l}0,015495 \\
0,014854 \\
0,019134\end{array}$ & $\begin{array}{l}\text { A } \\
\text { A } \\
\text { B }\end{array}$ \\
\hline $\begin{array}{l}\text { Richards } \\
\text { Richards }\end{array}$ & $\begin{array}{c}\text { Guzerá(Guzera) } \\
\text { Nelore (Nellore) } \\
\text { Nelore Mocho (Polled Nellore) }\end{array}$ & $\begin{array}{l}485,30 \\
487,58 \\
479,32\end{array}$ & $\begin{array}{l}\mathrm{A} \\
\mathrm{A} \\
\mathrm{A}\end{array}$ & $\begin{array}{l}0,0024578 \\
0,0023314 \\
0,0024318\end{array}$ & $\begin{array}{l}\mathrm{A} \\
\mathrm{A} \\
\mathrm{A}\end{array}$ \\
\hline $\begin{array}{l}\text { Von Bertalanffy } \\
\text { Von Bertalanffy }\end{array}$ & $\begin{array}{c}\text { Guzerá(Guzera) } \\
\text { Nelore (Nellore) } \\
\text { Nelore Mocho (Polled Nellore) }\end{array}$ & $\begin{array}{l}419,50 \\
447,28 \\
421,98\end{array}$ & $\begin{array}{l}\text { A } \\
\text { A } \\
\text { A }\end{array}$ & $\begin{array}{l}0,0035112 \\
0,0036707 \\
0,0041491\end{array}$ & $\begin{array}{l}\text { A } \\
\text { A } \\
\text { B }\end{array}$ \\
\hline $\begin{array}{l}\text { Brody } \\
\text { Brody }\end{array}$ & $\begin{array}{c}\text { Guzerá(Guzera) } \\
\text { Nelore (Nellore) } \\
\text { Nelore Mocho (Polled Nellore) }\end{array}$ & $\begin{array}{l}441,11 \\
487,48 \\
489,96\end{array}$ & $\begin{array}{l}\text { A } \\
\text { B } \\
\text { B }\end{array}$ & $\begin{array}{l}0,0022776 \\
0,0021402 \\
0,0023483\end{array}$ & $\begin{array}{l}\mathrm{A} \\
\mathrm{A} \\
\mathrm{A}\end{array}$ \\
\hline $\begin{array}{l}\text { Gompertz } \\
\text { Gompertz }\end{array}$ & $\begin{array}{c}\text { Guzerá(Guzera) } \\
\text { Nelore (Nellore) } \\
\text { Nelore Mocho (Polled Nellore) }\end{array}$ & $\begin{array}{l}405,41 \\
432,69 \\
403,75\end{array}$ & $\begin{array}{l}\text { A } \\
\text { A } \\
\text { A }\end{array}$ & $\begin{array}{l}0,0041959 \\
0,0045325 \\
0,0047726\end{array}$ & $\begin{array}{l}\text { A } \\
\text { B } \\
\text { B }\end{array}$ \\
\hline $\begin{array}{l}\text { Logístico } \\
\text { Logistic }\end{array}$ & $\begin{array}{c}\text { Guzerá(Guzera) } \\
\text { Nelore (Nellore) } \\
\text { Nelore Mocho (Polled Nellore) }\end{array}$ & $\begin{array}{l}401,35 \\
426,70 \\
385,38\end{array}$ & $\begin{array}{l}\text { A B } \\
\text { A } \\
\text { B }\end{array}$ & $\begin{array}{l}0,0070562 \\
0,0073056 \\
0,0084584\end{array}$ & $\begin{array}{l}\mathrm{A} \\
\mathrm{A} \\
\mathrm{B}\end{array}$ \\
\hline $\begin{array}{l}\text { Mitscherlich } \\
\text { Mitscherlich }\end{array}$ & $\begin{array}{c}\text { Guzerá(Guzera) } \\
\text { Nelore (Nellore) } \\
\text { Nelore Mocho (Polled Nellore) }\end{array}$ & $\begin{array}{l}416,83 \\
443,86 \\
436,51\end{array}$ & $\begin{array}{l}\text { A } \\
\text { A } \\
\text { A }\end{array}$ & $\begin{array}{l}0,0028457 \\
0,0027699 \\
0,0030677\end{array}$ & $\begin{array}{l}\mathrm{A} \\
\mathrm{A} \\
\mathrm{A}\end{array}$ \\
\hline
\end{tabular}

* Letras diferentes na mesma coluna, para o mesmo modelo, diferem a $5 \%(P<0,05)$ pelo teste Student-Newman-Keuls.

* Different characters in the same column, for the same model, differ at $5 \%(P<0.05)$ by Student-Newman-Keuls test. 
Tabela 12 - Qualidade de ajuste do modelo misto, pelo critério de informação de Akaike (AIC), valores preditos para os componentes de variância e suas significâncias

Table 12 - Mixed linear model quality adjust, by Akaike information criteria (AIC), variance components predicted values and its significance

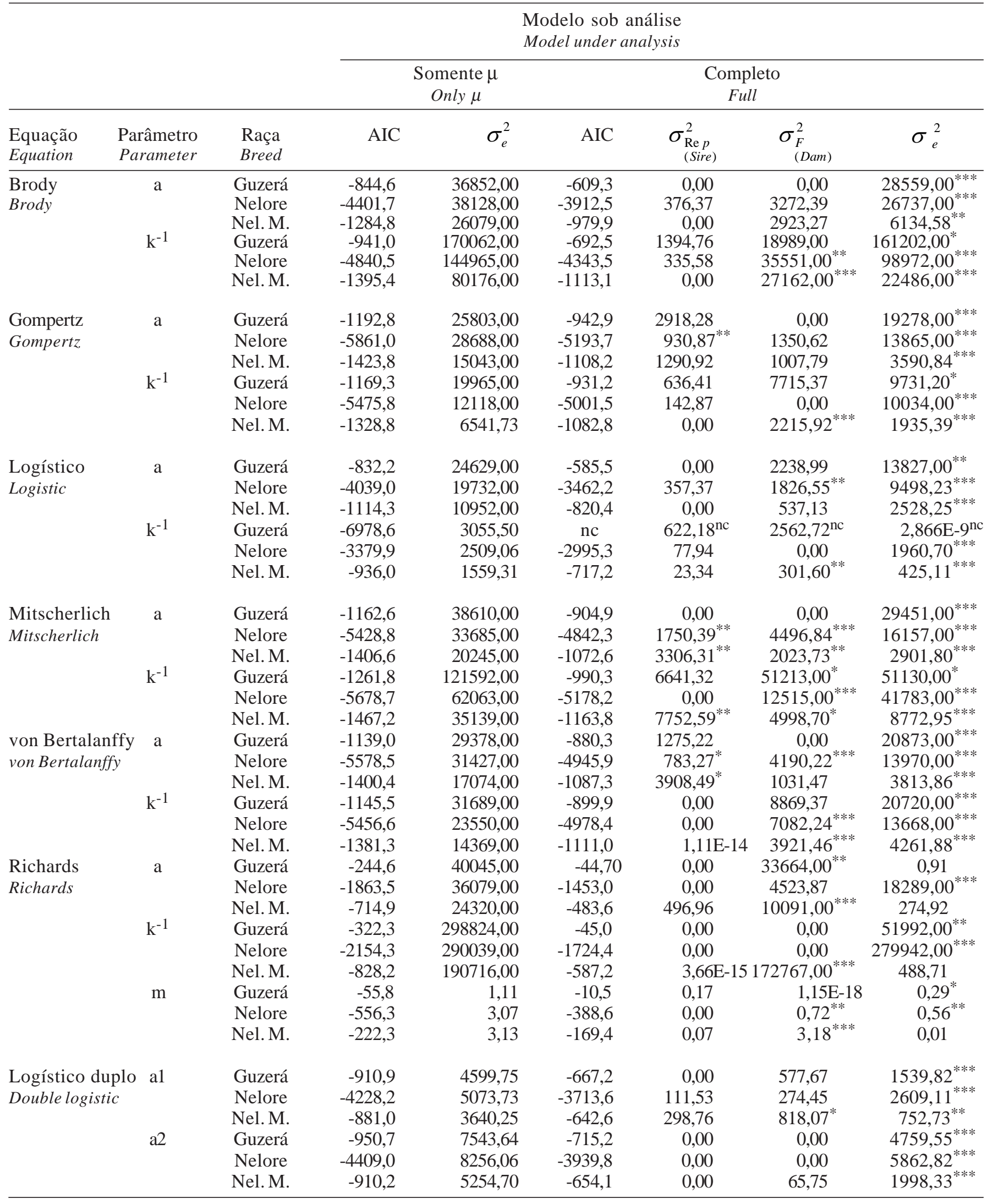




\begin{tabular}{|c|c|c|c|c|c|c|c|c|}
\hline \multirow[b]{3}{*}{$\begin{array}{l}\text { Equação } \\
\text { Equation }\end{array}$} & \multirow[b]{3}{*}{$\begin{array}{l}\text { Parâmetro } \\
\text { Parameter }\end{array}$} & \multirow[b]{3}{*}{$\begin{array}{l}\text { Raça } \\
\text { Breed }\end{array}$} & \multicolumn{5}{|c|}{$\begin{array}{l}\text { Modelo sob análise } \\
\text { Model under analysis }\end{array}$} & \\
\hline & & & \multicolumn{2}{|c|}{$\begin{array}{l}\text { Somente } \mu \\
\text { Only } \mu\end{array}$} & \multicolumn{4}{|c|}{$\begin{array}{l}\text { Completo } \\
\text { Full }\end{array}$} \\
\hline & & & AIC & $\sigma_{e}^{2}$ & AIC & $\sigma_{\text {Re } p}^{2}$ & $\sigma_{F}^{2}$ & $\sigma_{e}^{2}$ \\
\hline \multirow[t]{3}{*}{ b1 } & Guzerá & $-882,2$ & 3218,84 & $-673,4$ & $1020,85^{*}$ & 0,00 & $1801,36^{* * *}$ & \\
\hline & & Nelore & $-3813,5$ & 1662,03 & $-3449,8$ & 0,00 & $246,64^{*}$ & $1112,82^{* * *}$ \\
\hline & & Nel. M. & $-835,0$ & 2039,06 & $-626,8$ & $-644,82^{*}$ & $643,45^{*}$ & 442,46 \\
\hline \multirow[t]{9}{*}{ b2 } & Guzerá & $-1442,4$ & 3388200,00 & $-1114,7$ & 175244,00 & 303157,00 & $3158536,00^{* * *}$ & \\
\hline & & Nelore & $-4531,1$ & 11469,00 & $-4112,4$ & 312,97 & 0,00 & $9548,47^{* * *}$ \\
\hline & & Nel. M. & 990,6 & 14448,00 & $-746,4$ & 1780,84 & $5715,40^{* * *}$ & $3368,17^{* *}$ \\
\hline & $\mathrm{a} 1+\mathrm{a} 2$ & Guzerá & $-1013,2$ & 16390,00 & $-742,3$ & 0,00 & 0,00 & $7477,52^{* * *}$ \\
\hline & & Nelore & $-4697,7$ & 17956,00 & $-4127,1$ & 192,74 & 801,38 & $9258,10^{* * *}$ \\
\hline & & Nel. M. & $-975,1$ & 11878,00 & $-680,1$ & 815,24 & $1576,45^{* *}$ & $1288,43^{* *}$ \\
\hline & $\mathrm{k}$ & Guzerá & 563,9 & $0,51 \mathrm{E}-4$ & 401,8 & 9,97E-8 & 0,00 & $0,38 \mathrm{E}-4^{* * *}$ \\
\hline & & Nelore & 2928,9 & $0,19 E-4$ & 2627,1 & $9,33 \mathrm{E}-8$ & 7,33E-7 & $0,17 \mathrm{E}-4^{* * *}$ \\
\hline & & Nel. M. & 292,8 & $0,14 \mathrm{E}-2$ & 227,1 & 0,00 & $0,10 \mathrm{E}-2^{* * *}$ & $1,07 \mathrm{E}-7$ \\
\hline
\end{tabular}

${ }^{*} \mathrm{P}<0,10,{ }^{* *} \mathrm{P}<0,05,{ }^{* * *} \mathrm{P}<0,01$, Teste $\mathrm{Z}$.

${ }^{*} P<0.10,{ }^{* *} P<0.05,{ }^{* *} P<0.01, Z$ test.

Tabela 13 - Valor de $F$ para a influência dos efeitos fixos sobre os parâmetros estimados para as equações não-lineares Table 13 - $F$ values for fixed effects influence on non-linear equations parameters estimates

\begin{tabular}{|c|c|c|c|c|c|c|c|c|}
\hline \multirow[b]{2}{*}{$\begin{array}{l}\text { Modelo } \\
\text { Model }\end{array}$} & \multirow[b]{2}{*}{$\begin{array}{l}\text { Variável } \\
\text { Variable }\end{array}$} & \multirow[b]{2}{*}{$\begin{array}{l}\text { Raça } \\
\text { Breed }\end{array}$} & \multicolumn{6}{|c|}{$\begin{array}{l}\text { Efeito } \\
\text { Effect }\end{array}$} \\
\hline & & & $\begin{array}{l}\text { Sexo } \\
\text { Sex }\end{array}$ & $\begin{array}{l}\text { Prop } \\
\text { Herd }\end{array}$ & $\begin{array}{c}\text { Ano nasc } \\
\text { Year }\end{array}$ & $\begin{array}{l}\text { Mes nasc } \\
\text { Month }\end{array}$ & $\begin{array}{l}\text { Regim } \\
\text { Manag. }\end{array}$ & $\begin{array}{l}\text { Regim*Sexo* } \\
\text { Sex Manag. }\end{array}$ \\
\hline \multirow[t]{6}{*}{ Brody } & \multirow[t]{3}{*}{ a } & Guzerá & 1,83 & 2,28 & 0,87 & 1,26 & 0,37 & 0,03 \\
\hline & & Nelore & 2,79 & 1,90 & $2,64^{*}$ & 1,60 & 1,96 & 0,03 \\
\hline & & Nel. M. & $35,03^{* * *}$ & 3,64 & $5,05^{*}$ & 2,73 & $6,94^{* *}$ & 0,63 \\
\hline & \multirow[t]{3}{*}{$\mathrm{k}^{-1}$} & Guzerá & 2,21 & 1,24 & 0,66 & 0,79 & 2,37 & 0,38 \\
\hline & & Nelore & 0,58 & 1,02 & $2,53^{*}$ & $2,70^{*}$ & 2,26 & 0,12 \\
\hline & & Nel. M. & $7,50^{* *}$ & 2,81 & 2,66 & 1,65 & 1,90 & 0,12 \\
\hline \multirow[t]{6}{*}{ Gompertz } & \multirow[t]{3}{*}{ a } & Guzerá & 4,61 & 1,86 & 0,46 & 0,95 & 3,91 & 0,10 \\
\hline & & Nelore & $28,62^{* * *}$ & $2,97^{* * *}$ & $5,97^{* * *}$ & 0,97 & $12,57^{* * *}$ & 1,72 \\
\hline & & Nel. M. & $56,20^{* * *}$ & 2,08 & 3,22 & 2,31 & $10,03^{* *}$ & 1,10 \\
\hline & \multirow[t]{3}{*}{$\mathrm{k}^{-1}$} & Guzerá & 1,77 & 2,02 & 1,10 & 0,72 & 5,60 & 0,52 \\
\hline & & Nelore & $5,80^{* *}$ & 1,25 & $4,61^{* * *}$ & 1,61 & $3,12^{* *}$ & 0,59 \\
\hline & & Nel. M. & $14,50^{* *}$ & 2,17 & $3,60^{*}$ & 2,69 & 3,70 & 0,00 \\
\hline \multirow[t]{6}{*}{ Logístico } & \multirow[t]{3}{*}{ a } & Guzerá & 11,09 & 1,58 & 1,03 & 0,67 & 6,14 & 0,12 \\
\hline & & Nelore & $36,40^{* * *}$ & $2,53^{*}$ & $4,09^{* *}$ & 1,08 & $8,84^{* * *}$ & 0,66 \\
\hline & & Nel. M. & $39,27^{* * *}$ & 3,69 & 2,62 & 3,64 & $17,88^{* *}$ & 2,12 \\
\hline & \multirow[t]{3}{*}{$\mathrm{k}^{-1}$} & Guzerá & nc & nc & nc & nc & nc & nc \\
\hline & & Nelore & $7,93^{* *}$ & 1,68 & $2,90^{*}$ & 1,83 & $4,60^{* *}$ & 0,63 \\
\hline & & Nel. M. & $17,89^{* *}$ & 2,87 & 3,58 & $6,46^{*}$ & 4,02 & 0,19 \\
\hline \multirow[t]{6}{*}{ Mitscherlich } & \multirow[t]{3}{*}{$\mathrm{a}$} & Guzerá & 0,53 & 2,52 & 0,98 & 1,82 & 4,80 & 0,13 \\
\hline & & Nelore & $10,33^{* * *}$ & $3,99^{* * *}$ & $2,96^{* * *}$ & 1,70 & $10,21^{* * *}$ & 0,40 \\
\hline & & Nel. M. & $32,15^{* * *}$ & 3,09 & 3,32 & 2,19 & $11,74^{* *}$ & 0,23 \\
\hline & \multirow[t]{3}{*}{$\mathrm{k}^{-1}$} & Guzerá & 0,22 & 3,11 & 1,76 & 1,29 & 0,55 & 0,21 \\
\hline & & Nelore & $4,44^{* *}$ & $1,96^{* *}$ & $3,40^{* * *}$ & 0,98 & 1,07 & 2,14 \\
\hline & & Nel. M. & $5,43^{*}$ & 1,99 & 1,74 & 2,15 & 0,03 & 1,12 \\
\hline
\end{tabular}


Continuação da Tabela $13 . .$.

\begin{tabular}{|c|c|c|c|c|c|c|c|c|}
\hline \multirow{2}{*}{$\begin{array}{l}\text { Modelo } \\
\text { Model }\end{array}$} & \multirow{2}{*}{$\begin{array}{l}\text { Variável } \\
\text { Variable }\end{array}$} & \multirow{2}{*}{$\begin{array}{l}\text { Raça } \\
\text { Breed }\end{array}$} & \multicolumn{6}{|c|}{$\begin{array}{l}\text { Efeito } \\
\text { Effect }\end{array}$} \\
\hline & & & Sexo & Prop & Ano nasc & Mes nasc & Regim & Regim*Sexo* \\
\hline \multirow[t]{4}{*}{ von Bertalanffy } & y a & Guzerá & 5,19 & 2,38 & 0,74 & 1,14 & 3,54 & 0,08 \\
\hline & & Nelore & $36,33^{* * *}$ & $3,28^{* * *}$ & $4,38^{* * *}$ & $1,80^{*}$ & $6,40^{* * *}$ & 0,07 \\
\hline & & Nelore & $7,68^{* * *}$ & 1,09 & $4,39^{* * *}$ & $1,97^{*}$ & $4,31^{* *}$ & 0,53 \\
\hline & & Nel. M. & $6,37^{*}$ & 2,45 & 3,42 & 3,75 & 4,32 & 2,36 \\
\hline \multirow[t]{5}{*}{ Richards } & $\mathrm{a}$ & Guzerá & 0,01 & 0,93 & 0,88 & 0,92 & 1,97 & 0,41 \\
\hline & & Nelore & 1,42 & 2,13 & 1,23 & 1,19 & 3,51 & 0,84 \\
\hline & $\mathrm{m}$ & Guzerá & 0,31 & 0,98 & 2,82 & 1,84 & 0,39 & 1,50 \\
\hline & & Nelore & 0,32 & $15,45^{* *}$ & 0,66 & 0,86 & 0,17 & 0,18 \\
\hline & & Nel. M. & 1,56 & 6,54 & 3,41 & 3,04 & 0,66 & 1,48 \\
\hline \multirow[t]{10}{*}{ Logístico duplo } & a1 & Guzerá & 0,25 & 3,39 & 4,23 & 1,64 & 18,37 & 0,14 \\
\hline & & Nelore & 1,52 & $4,03^{* * *}$ & $2,94^{* *}$ & $2,85^{* *}$ & $38,48^{* * *}$ & 1,54 \\
\hline & & Nel. M. & 8,43 & 1,58 & 2,30 & 1,14 & 7,20 & 0,62 \\
\hline & $\mathrm{a} 2$ & Guzerá & 10,18 & 4,86 & 0,98 & 1,70 & 5,49 & 1,94 \\
\hline & & Nelore & $14,82^{* * *}$ & $3,23^{* *}$ & $3,41^{* *}$ & $2,29^{*}$ & $4,85^{* *}$ & 0,16 \\
\hline & & Nel. M. & $33,24^{* *}$ & 0,93 & 2,23 & 2,11 & 2,48 & 0,41 \\
\hline & b1 & Guzerá & 0,00 & 2,19 & 1,53 & 2,39 & 4,87 & 2,30 \\
\hline & $\mathrm{k}$ & Guzerá & 1,78 & 4,14 & 1,17 & 1,46 & 3,57 & 0,77 \\
\hline & & Nelore & 0,37 & 1,31 & 1,32 & $3,52^{* *}$ & 2,42 & 1,75 \\
\hline & & Nel. M. & 0,27 & 5,49 & 8,28 & 38,79 & 13,00 & 14,19 \\
\hline
\end{tabular}

nc Algoritmo não convergiu.

${ }^{n c}$ Algoritm not converged.

${ }^{*} \mathrm{P}<0,10$, ${ }^{* *} \mathrm{P}<0,05$, ${ }^{* *} \mathrm{P}<0,01$, Teste $F$; - Sem possibilidade de realizar o teste $F$.

* $P<0,10$, ** $P<0,05$, *** $P<0,01, F$ test; - Without possibility to use $F$ test.

Em termos gerais, os efeitos fixos que mais influenciaram os parâmetros estimados para as equações não-lineares, em ordem de importância, foram sexo, propriedade, ano e mês de nascimento e regime alimentar, com, respectivamente, 23, 18, 16, 11 e 11 valores significativos (Tabela 13). A interação de sexo*sistema de criação influenciou apenas o parâmetro $b_{1}$ do modelo Logístico Duplo, para a raça Nelore. Os parâmetros estimados pelo modelo de Richards sofreram pouca influência dos efeitos fixos, demonstrando pouca variabilidade, e foram seguidos do modelo Brody. Os modelos de maior variabilidade foram o Logístico Duplo e o von Bertalanffy. 


\section{Conclusões}

A análise dos dados de crescimento corporal dos animais por meio de modelos não-lineares depende diretamente da correta observação e do acompanhamento de observações de peso-idade, de modo que a falta de observações em idades mais avançadas pode prejudicar as estimativas da curva e seus parâmetros. A verificação da convergência da equaçãonão garantiu que os parâmetros estimados se mantivessem nos limites biologicamente aceitáveis. Portanto, deveriam ser observados criteriosamente quais seriam passíveis de uma análise, para estudo da influência de efeitos fixos e aleatórios. O modelo logístico duplo foi considerado o mais satisfatório computacionalmente, enquanto os modelos de Richards e o Brody, os melhores biologicamente.

A influência dos efeitos fixos colaborou com a noção de que o manejo e a correta nutrição poderiam influenciar o desenvolvimento corporal, além dos componentes genéticos. A variabilidade dos efeitos aleatórios possibilitaria o planejamento da modificação da curva de crescimento por meio da exploração de indivíduos mais qualificados, selecionados a partir de um programa de melhoramento genético.

\section{Literatura Citada}

BATSCHELET, E. Introdução à matemática para biocientistas. São Paulo: EDUSP, 1984. 596p.

von BERTALANFFY, L. Quantitative laws in metabolism and growth. Quarterly Review of Biology, v.32, p.218, 1957.

BRODY, S. Bioenergetics and growth. New York: Rheinhold Pub. Corp., 1945. 645p.

BROWN, J.E.; FITZHUGH JR., H.A.; CARTWRIGHT, T.C. A comparison of nonlinear models for describing weight-age relationships in cattle. Journal of Animal Science, v.42, n.4, p.810-818, 1976.

DOREN, P.E.; BAKER, J.F.; LONG, C.R. et al. Estimating parameters of growth curves of bulls. Journal of Animal Science, v.67, p.143-1445, 1989.

FITZHUGH JR., H.A. Analysis of growth curves and strategies for altering their shape. Journal of Animal Science, v.42, n.4, p.1036- 1051, 1976.

GOONEWARDENE, L.A.; BERG, R.T.; HARDIN, R.T. A growth study of beef cattle. Canadian Journal of Animal Science, v.61, p.1041-1048, 1981.

HOFFMAN, R.; VIEIRA, S. Uma introdução à econometria. 3.ed. São Paulo: HUCITEC. 1998. 379p.

KOENEN, E.P.C.; GROEN, A.F. Genetic analysis of growth patterns of black and white dairy heifers. Journal of Dairy Science, v.79, p.495-501, 1996.

KROLL, L.B.; TORNERO, M.T.T. Modelo de Gompertz com sazonalidade e autocorrelação nos erros para ajuste do crescimento ponderal em vaca leiteira. Pesquisa Agropecuária Brasileira, v.29, n.5, p.707-711, 1994.
KVÅLSETH, T.O. Cautionary note about $\mathrm{R}^{2}$. The American Statistician, parte 1, v.39, n.4, p.279-285, 1985.

LAWRENCE, T.L.J.; FOWLER, V.R. Growth of farm animals. New York: CAB International, 1997. 330p.

LUDWIG, A.; SILVA, M.A.; OLIVEIRA, L.M. Ajustamento de modelos estatísticos exponenciais ao crescimento de gado nelore. Pesquisa Agropecuária Brasileira, v.16, n.2, p.297302, 1981.

MAZZINI, A.R.A. Análise da curva de crescimento de machos Hereford considerando heterogeneidade de variâncias e autocorrelação dos erros. Lavras: Universidade Federal de Lavras, 2001. 94p. Dissertação (Mestrado em Experimentação Agrícola) - Universidade Federal de Lavras, 2001.

NELDER, J.A. The fitting of a generalization of the logistic curve. Biometrics, v.17, p.89-94, 1961.

NOBRE, P.R.C.; ROSA, A.N.; SILVA, L.O.C. et al. Curvas de crescimento de gado nelore ajustadas para diferentes frequências de pesagens. Pesquisa Agropecuária Brasileira, v.22, n.9/10, p.1027-1037, 1987.

OLIVEIRA, H.N.; LÔBO, R.B.; PEREIRA, C.S. Comparação de modelos não-lineares para descrever o crescimento de fêmeas da raça Guzerá. Pesquisa Agropecuária Brasileira, v.35, n.9, p.1843-1851, 2000.

PEARSON, A.M.; DUTSON, T.R. Growth regulation in farm animals. London: Elsevier Applied Science, 1991. 629p.

PEROTTO, D.; CUE, R.I.; LEE, A.J. Comparison of nonlinear functions for describing the growth curve of three genotypes of dairy catle. Canadian Journal of Animal Science, v.72, p.773-782, 1992.

PEROTTO, D.; CUE, R.I.; LEE, A.J. et al. Additive and nonadditive genetic effects of growth-curve parameters of Holstein, Ayrshire and crossbreed females. Canadian Journal of Animal Science, v.74, p.401-409, 1994.

PEROTTO, D.; CASTANHO, M.J.P.; CUBAS, A.C. et al. Efeitos genéticos sobre as estimativas dos parâmetros das curvas de crescimento de fêmeas bovinas Gir, Holandês x Gir e Holandês x Guzerá. Revista Brasileira de Zootecnia, v.26, n.4, p.719-725, 1997.

RICHARDS, F.J. A flexible growth function for empirical use. Journal of Experimental Botany, v.10, n.29, p.290-300, 1959.

ROSA, A.N.; SILVA, M.A.; MILAGRES, J.C. et al. Mudança da curva de crescimento de animais da raça Nelore mediante uso de índices de selção. Revista Brasileira de Zootecnia, v.8, n.4, p.610-621, 1979.

RUTLEDGE, J.J.; ROBISON, O.W.; EISEN, E.J. et al. Dynamics of genetic and maternal effects in mice. Journal of Animal Science, v.35, p.911-915, 1972.

STATISTICAL ANALYSES SYSTEM - SAS. SAS/STAT User's guide. Version 8.2. 4.ed. v.2. Cary: 2000.

TALLIS, G.M. Selection for na optimum growth curve. Biometrics, v.24, n.1, p.169-177, 1968.

TRENKLE, A.; MARPLE, D.N. Growth and development of meat animals. Journal of Animal Science, v.57, supl.2, p.273-283, 1983.

WINSOR, C.P. The Gompertz curve as a growth curve. Proceedings of the National Academy of Science, v.18, p.1-17, 1932.

Recebido em: 13/04/04

Aceito em: 15/07/05 